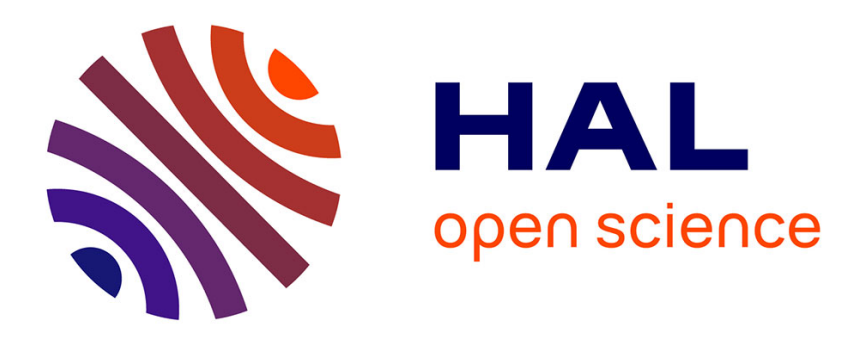

\title{
Sur la détermination de la valeur du champ magnétique terrestre au moyen du fluxmètre
}

\author{
M. Salazar
}

\section{To cite this version:}

M. Salazar. Sur la détermination de la valeur du champ magnétique terrestre au moyen du fluxmètre. J. Phys. Theor. Appl., 1913, 3 (1), pp.124-125. 10.1051/jphystap:019130030012400 . jpa-00241827

\section{HAL Id: jpa-00241827 https://hal.science/jpa-00241827}

Submitted on 1 Jan 1913

HAL is a multi-disciplinary open access archive for the deposit and dissemination of scientific research documents, whether they are published or not. The documents may come from teaching and research institutions in France or abroad, or from public or private research centers.
L'archive ouverte pluridisciplinaire HAL, est destinée au dépôt et à la diffusion de documents scientifiques de niveau recherche, publiés ou non, émanant des établissements d'enseignement et de recherche français ou étrangers, des laboratoires publics ou privés. 
SUR LA DÉTERMINATION DE LA VALEUR

DU GHAMP MAGNÉTIQUE TERRESTRE AU MOYEN DU FLUXMÈTRE;

Par M. SALAZAR.

La mesure du champ magnétique terrestre peut s'effectuer d'une manière à la fois simple et rapide par l'empìoi du fluxmètre Grassot.

Supposons qu'il s'agisse de déterminer, par exemple, la composante horizontale $\mathrm{H}$ : on reliera au fluxmètre les deux extrémités d'un fil de cuivre bobiné sur un cadre de manière à constituer un certain nombre de spires dont la surface totale $\mathrm{S}$ doit être connue exactement.

Ce cadre étant disposé verticalement, dans un plan perpendiculaire à la direction de la composante horizontale, est traversé par un flux ayant pour valeur :

$$
\varphi=\mathrm{SH} \text {. }
$$

Par une rotation d'un quart de tour, on amène le cadre dans le plan du méridien magnétique : le flux embrassé devient ainsi nul, et sa variation, égale à $\mathrm{SH}$, est indiquée par l'aiguille du fluxmètre. La valeur de $H$ s'en déduit immédiatement.

Pour augmenter la déviation, en vue d'obtenir une précision plus grande, on peut munir le cadre d'un collecteur redresseur à coquilles (cerceau de Delezenne) et le faire alors tourner d'un nombre de tours $n$ aussi grand que l'on veut. A chaque tour complet correspond un flux coupé égal à $4 \mathrm{SH}$. Pour $n$ tours, ce flux deviendra :

$$
\Phi=4 n \mathrm{SH}, \quad \text { d'où } \quad \mathrm{H}=\frac{\Phi}{4 n \mathrm{~S}},
$$

et la déviation de l'aiguille du fluxmètre sera $4 n$ fois plus grande que précédemment. L'un des principaux avantages de cette méthode, c'est que la vitesse de rotation peut être pour ainsi dire quelconque, et qu'il est inutile de la mesurer; toute la détermination se réduit à faire faire au cadre mobile un nombre de tours connu.

Dans une des expériences faites au laboratoire d'électrotechnique de Santiago (Chili), j'ai ainsi obtenu :

$$
\Phi=52 \times 10^{4} \text { (52 divisions des fluxmètres), }
$$


GOMPTES RENDUS DE L'ACADÉMIE DES SGIEXGES 125 pour :

$$
\begin{aligned}
& n=20 \text { tours } \\
& \mathrm{S}=302,8 \mathrm{~cm}^{2} \times 8+\text { spires },
\end{aligned}
$$

d'où :

$$
\mathrm{H}=0,23 \mathrm{~s} \text { gauss. }
$$

Des mesures comparatives faites en équilibrant au potentiomètre la f. é. m. développée par un cadre de 1.200 tours tournant à une vitesse connue, dans le même champ, ont donné pour $\mathrm{H}$ des valeurs constamment comprises entre 0,23 et 0,26 , justifiant ainsi la méthode que je viens d'indiquer. 